\title{
Synthesis and Redox Properties of Superbenzene Porphyrin Conjugates
}

Kharu Nisa,${ }^{\dagger \S}$ Vikas Khatri, ${ }^{\ddagger \S}$ Sharvan Kumar, ${ }^{\ddagger}$ Smriti Arora,${ }^{\dagger}$ Sohail Ahmad, ${ }^{\dagger}$ Anshu Dandia, ${ }^{\dagger}$ M.Thirumal, ${ }^{\dagger}$ Hemant K. Kashyap, ${ }^{* \star}$ and Shive M. S. Chauhan* ${ }^{\dagger}$

${ }^{\dagger}$ Department of Chemistry, University of Delhi, New Delhi 110007, India.

'Department of Chemistry, Indian Institute of Technology Delhi, Hauz Khas, New Delhi 110016, India.

E-mail: smschauhan@ chemistry.du.ac.in

hkashyap@chemistry.iitd.ac.in 


\section{Experimental Section}

Synthesis of Precursor hexaphenylbenzaldehyde (HPB-CHO):

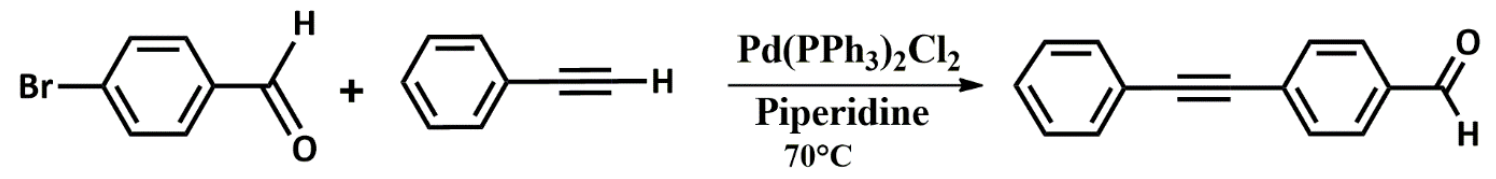

(1)

(2)
Tolan(3)

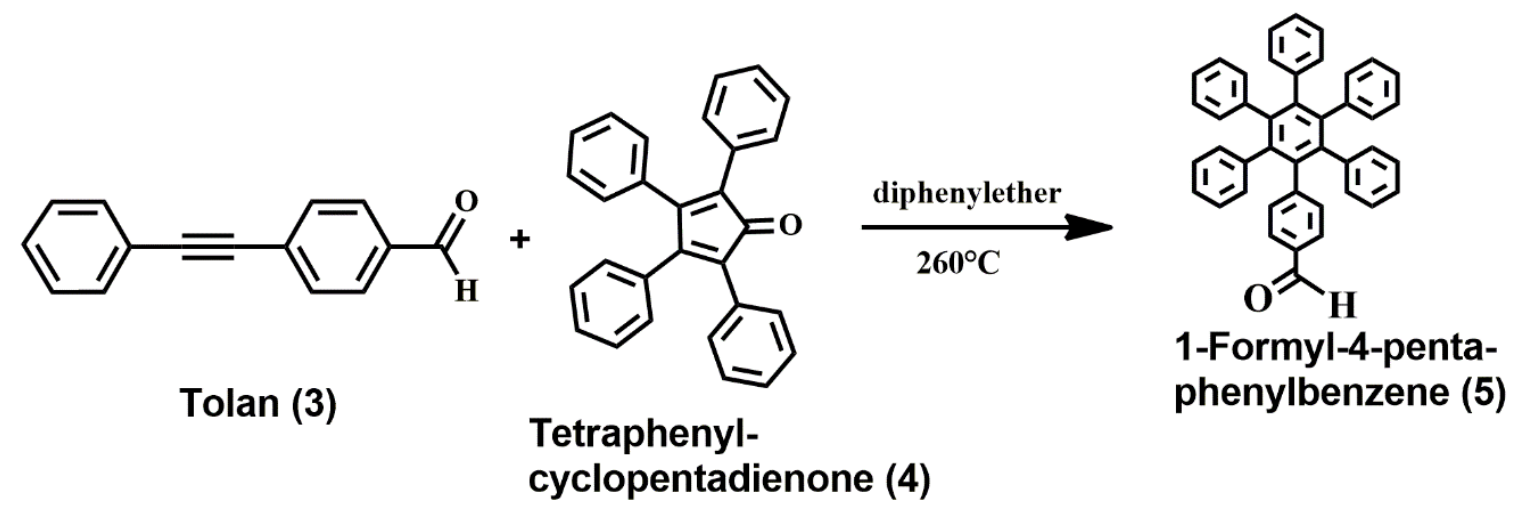

Figure S1. Scheme for the synthesis of precursor Hexaphenylbenzaldehyde (HPB-CHO) for porphyrin conjugates. 


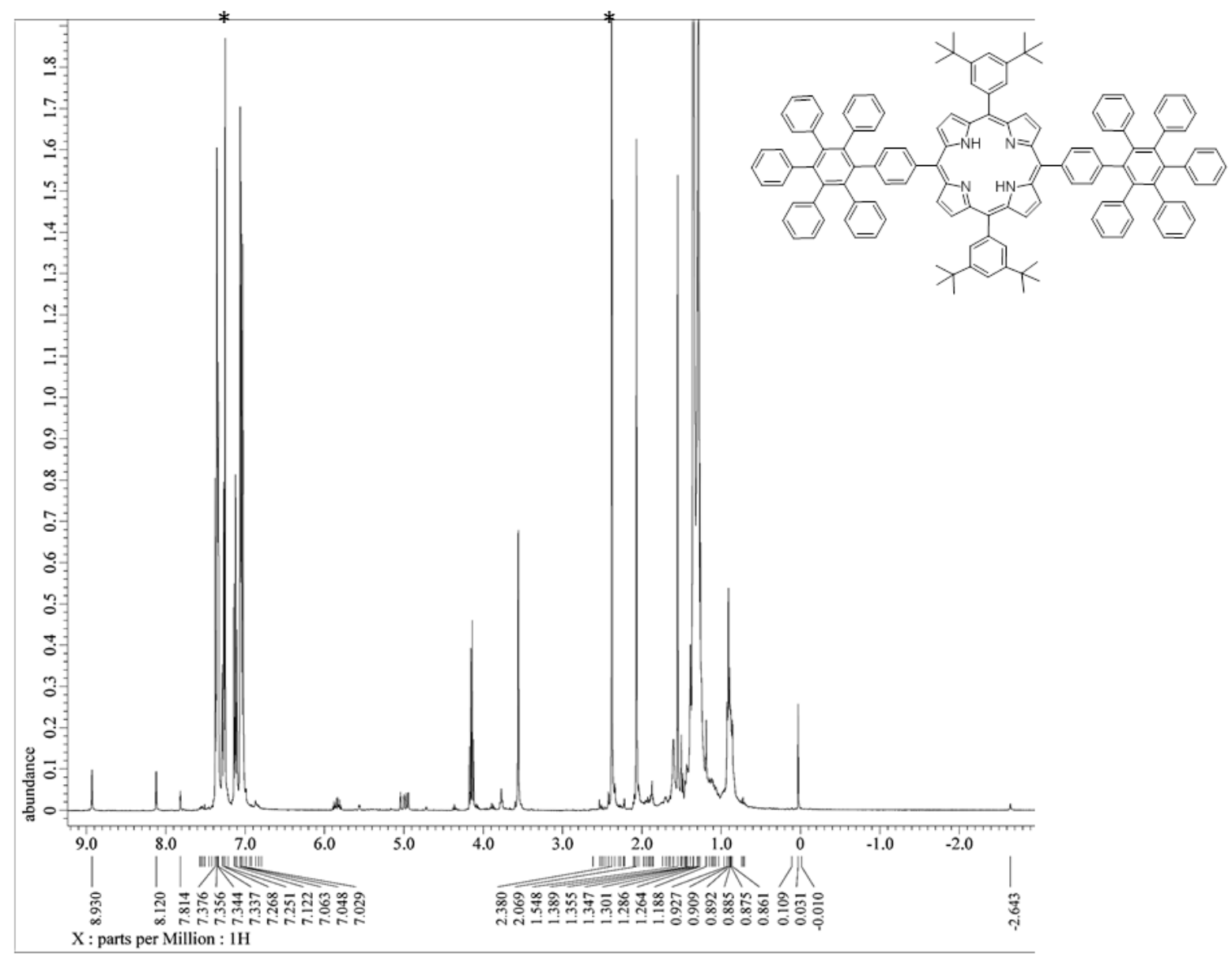

Figure S2. ${ }^{1} \mathrm{H} \mathrm{NMR}$ of 1 a porphyrin in $\mathrm{CDCl}_{3}$. 


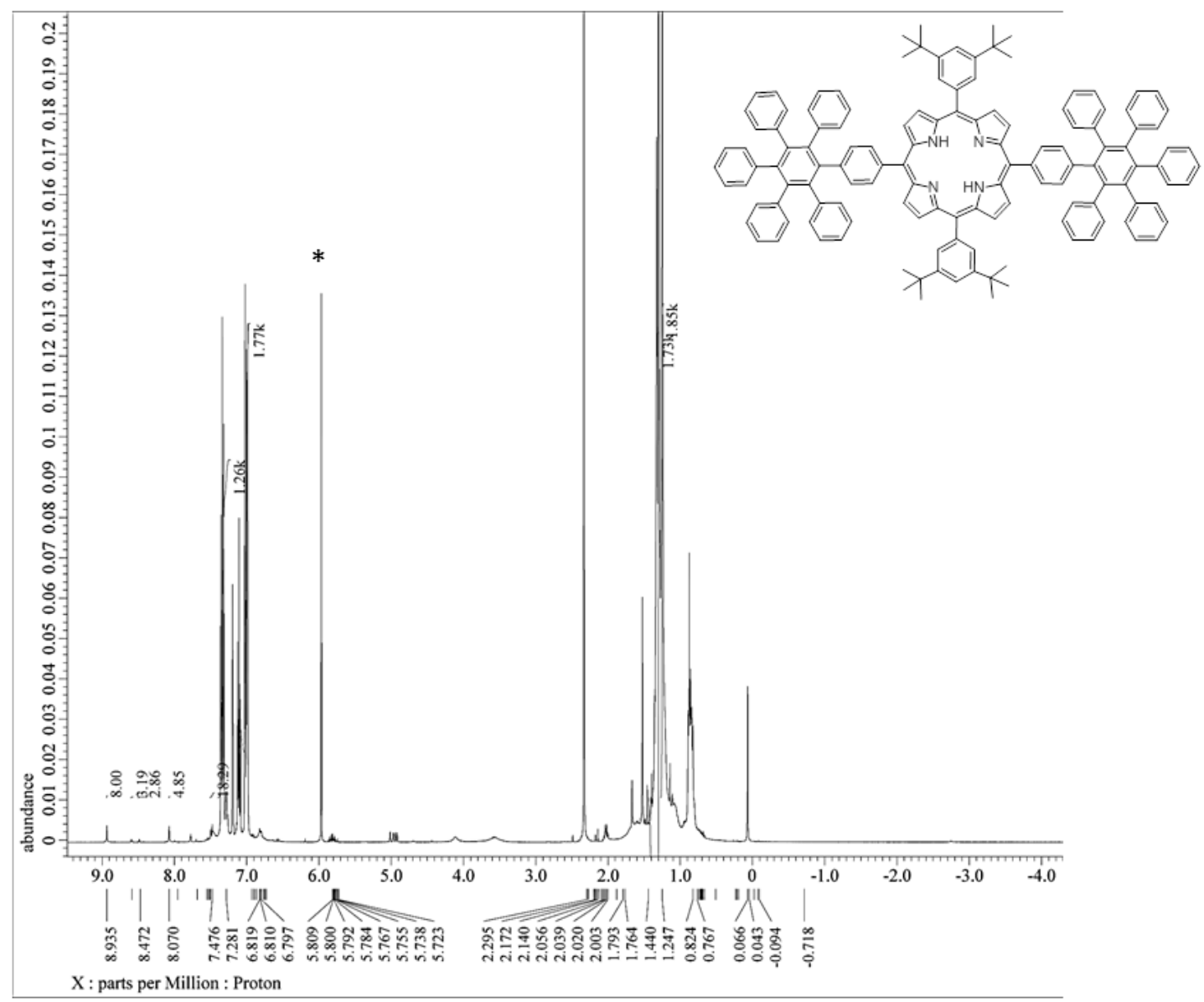

Figure S3. ${ }^{1} \mathrm{H}-\mathrm{NMR}$ of $1 \mathrm{a}$ in $\mathrm{C}_{2} \mathrm{D}_{2} \mathrm{Cl}_{4}$ (the same spectra when carried out in $\mathrm{CDCl}_{3}$ shows peak at the -2.64ppm). It depicts that two core $\mathrm{N}-\mathrm{H}$ protons are exchanging with two deuterium atoms. 


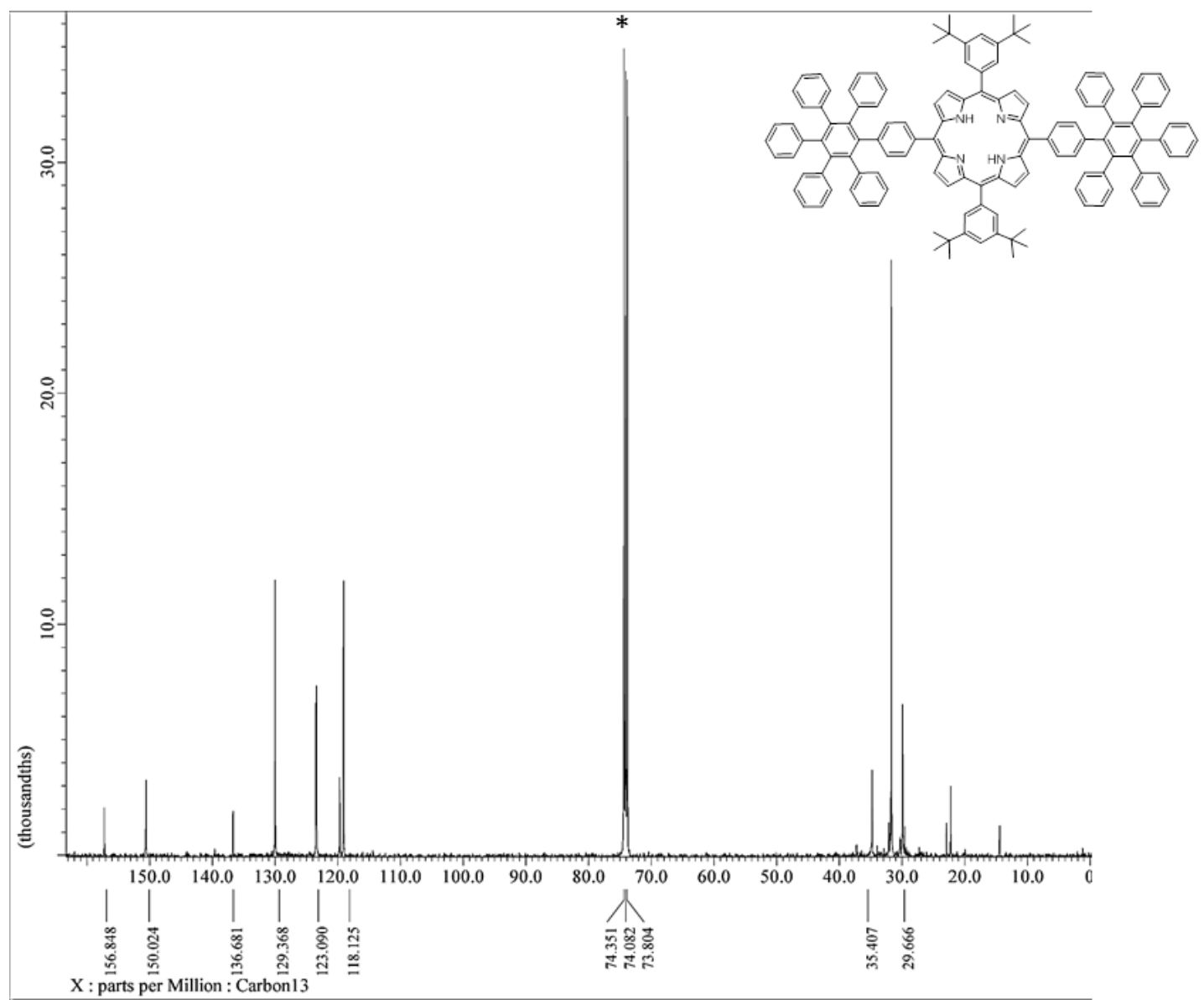

Figure S4. ${ }^{13} \mathrm{C}-\mathrm{NMR}$ of $1 \mathrm{a}$ porphyrin. 


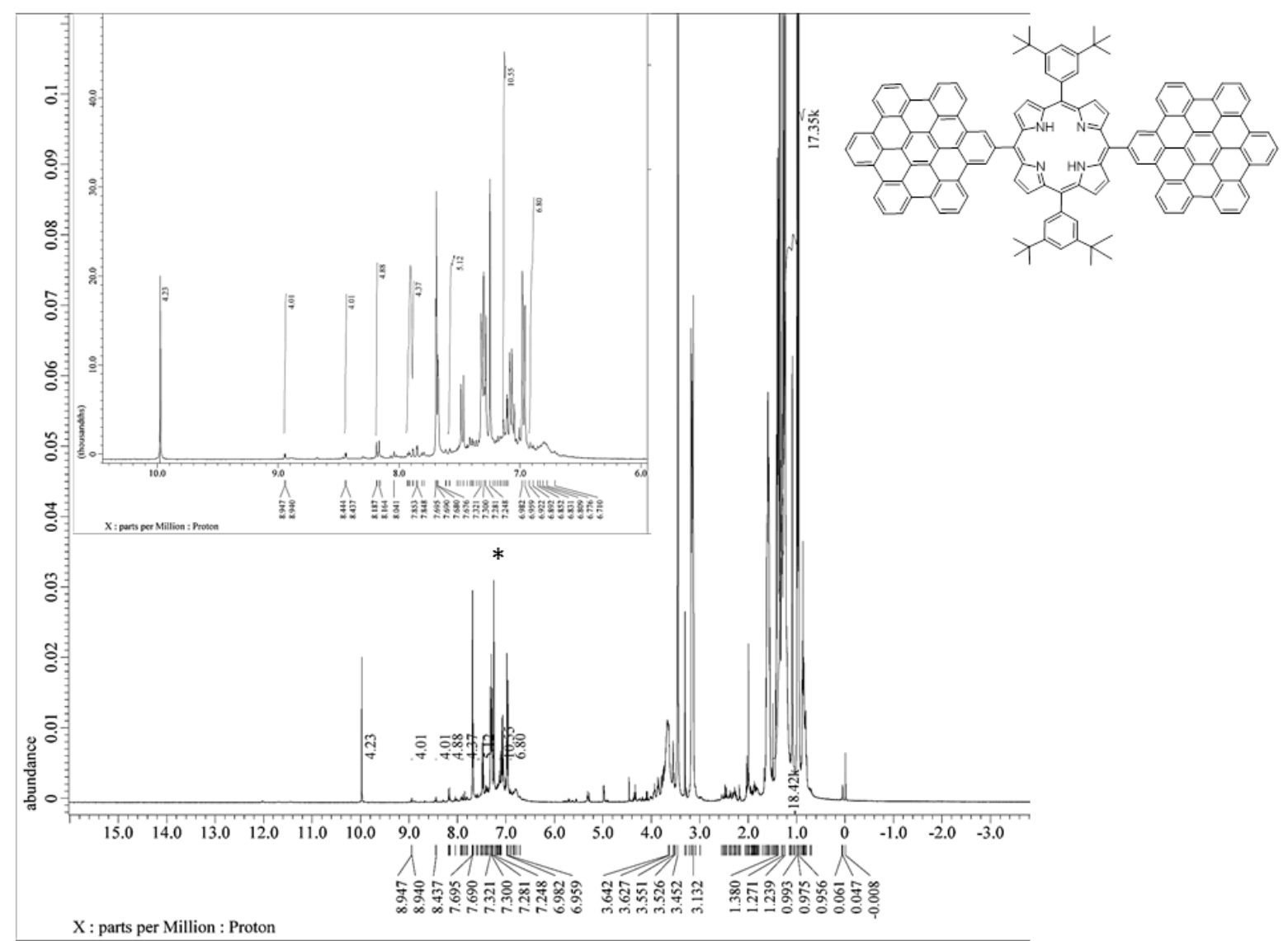

Figure S5. ${ }^{1} \mathrm{H}-\mathrm{NMR}$ of $\mathbf{1 b}$ porphyrin in $\mathrm{CDCl}_{3}+\mathrm{CS}_{2}$. 
$*$

*

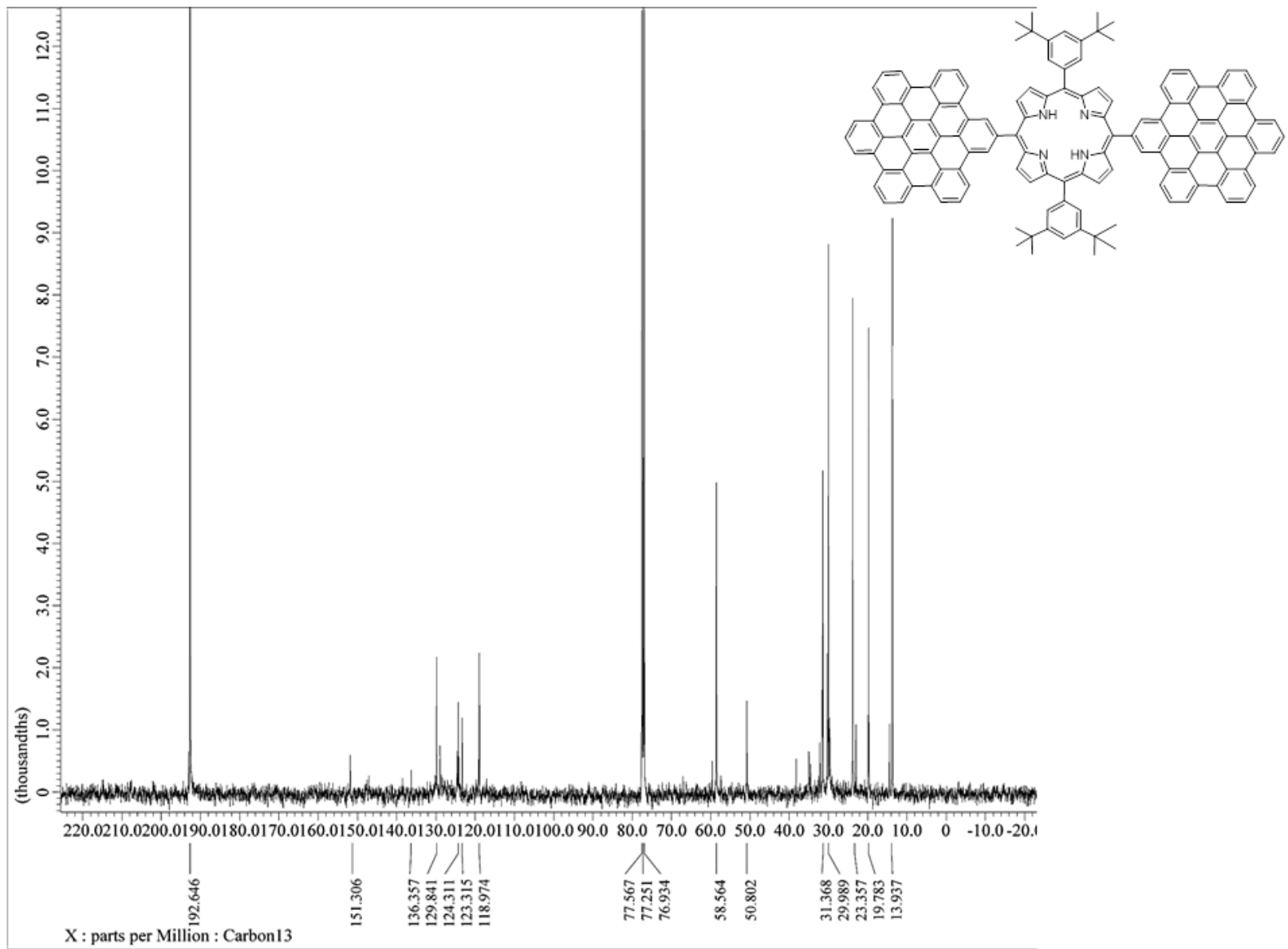

Figure S6. ${ }^{13} \mathrm{C}-\mathrm{NMR}$ spectra of $\mathbf{1 b}$ porphyrin in $\mathrm{CDCl}_{3}+\mathrm{CS}_{2}$. 


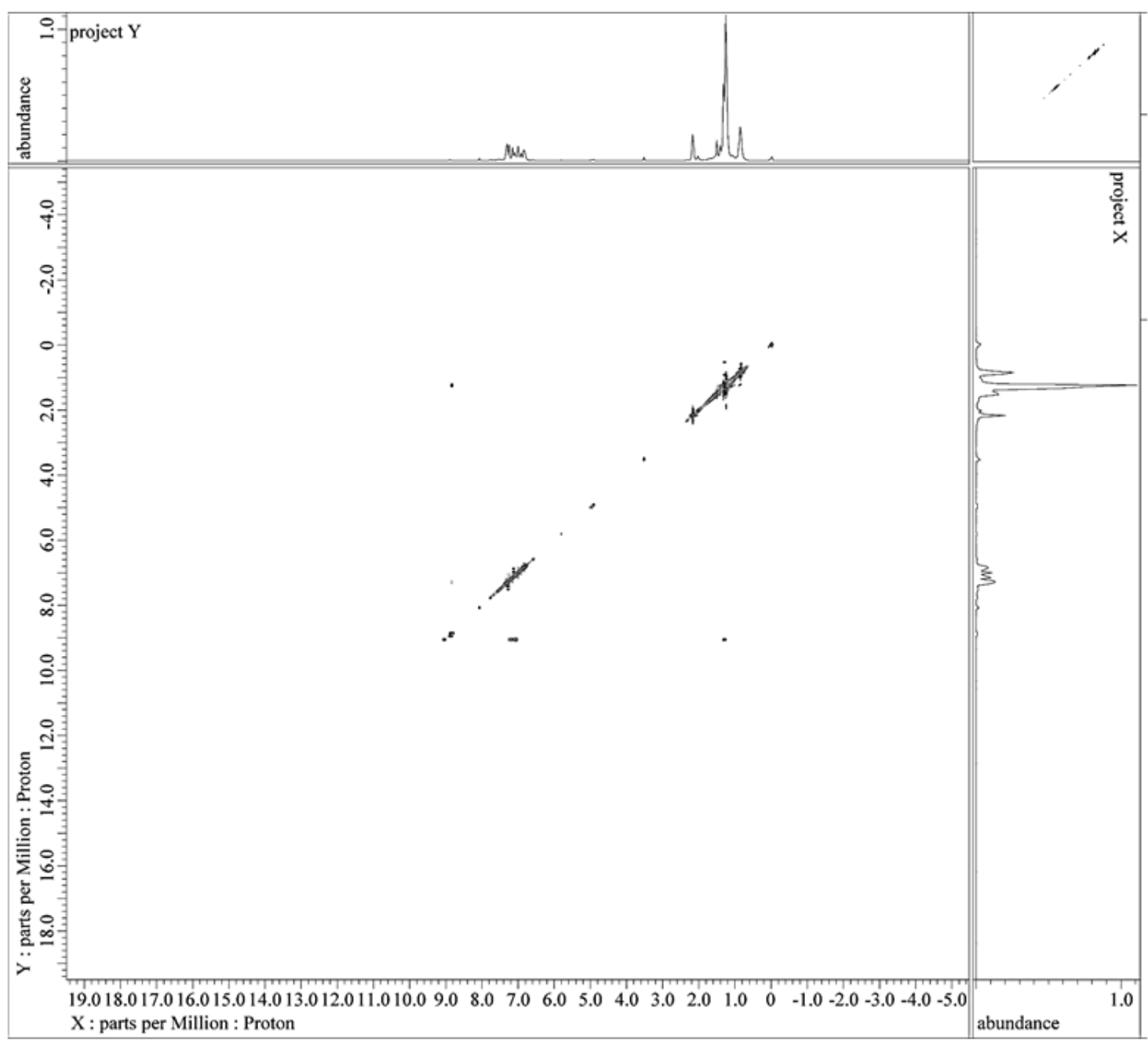

Figure S7. ${ }^{1} \mathrm{H}-{ }^{1} \mathrm{H}$ Noesy spectrum of $\mathbf{1 b}$. 


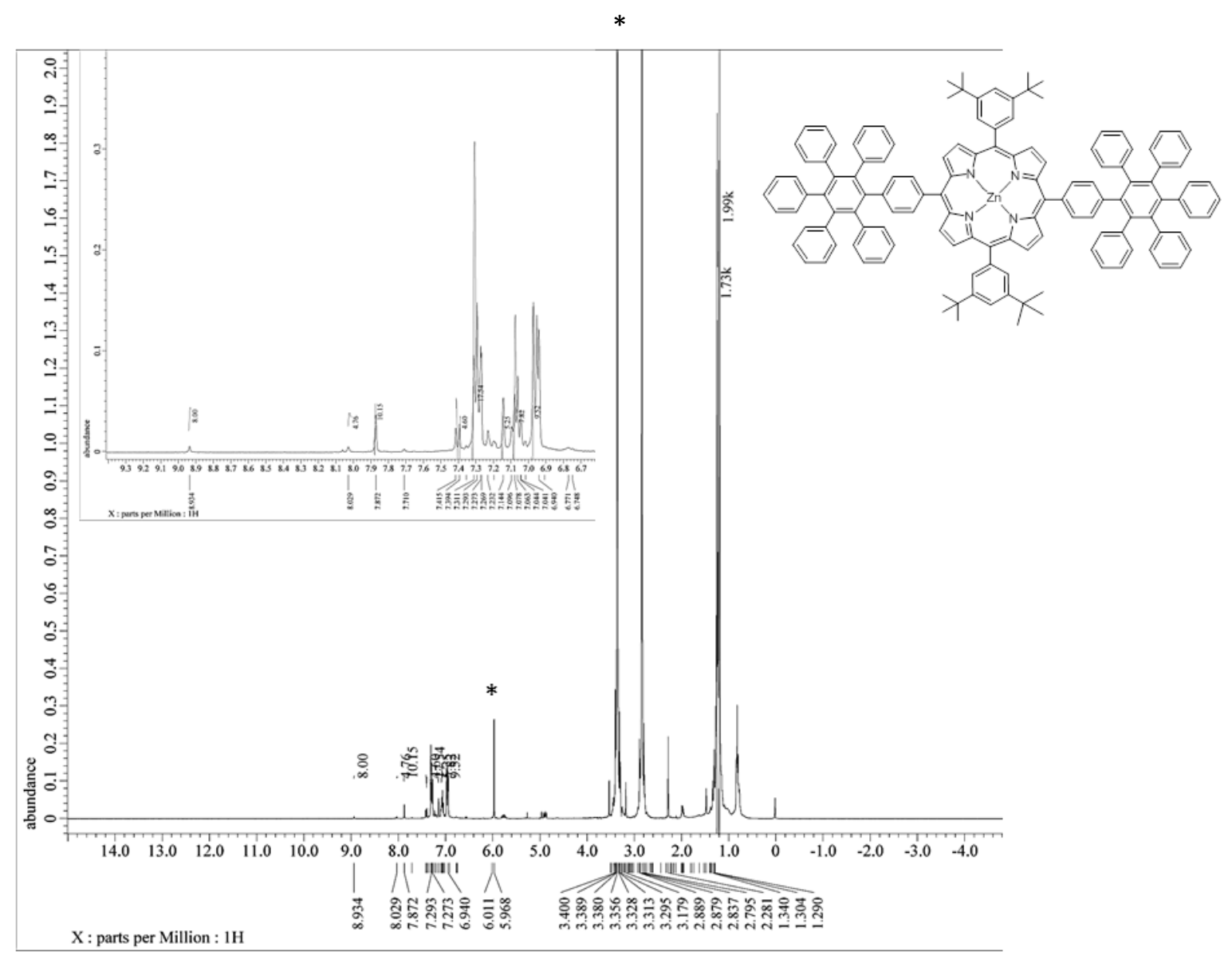

Figure S8. ${ }^{1} \mathrm{H}-\mathrm{NMR}$ of $1 \mathrm{c}$ porphyrin in $\mathrm{C}_{2} \mathrm{D}_{2} \mathrm{Cl}_{4}$. 


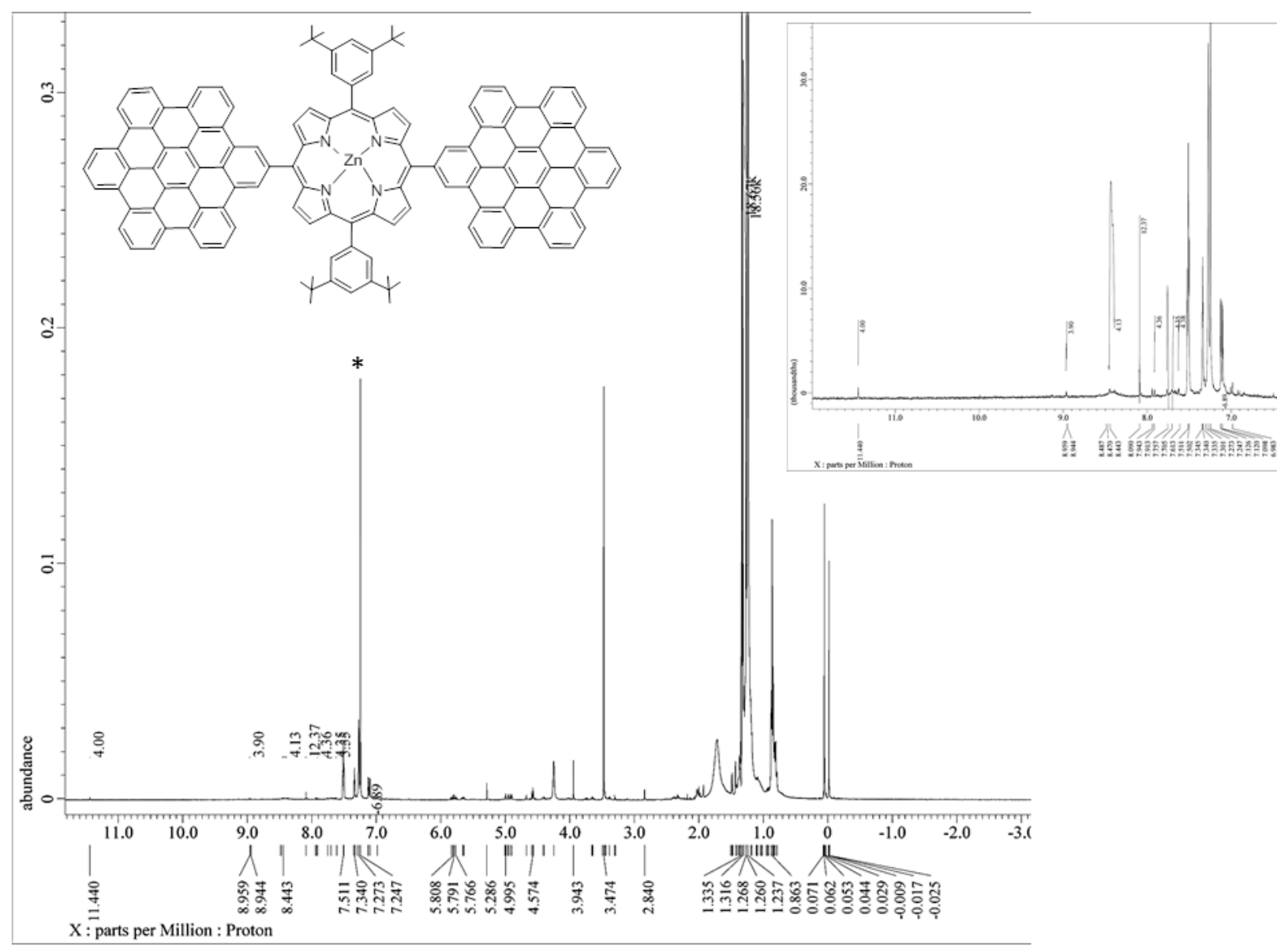

Figure S9. ${ }^{1} \mathrm{H}-\mathrm{NMR}$ of $\mathbf{1 d}$ porphyrin in $\mathrm{CDCl}_{3}$. 
*

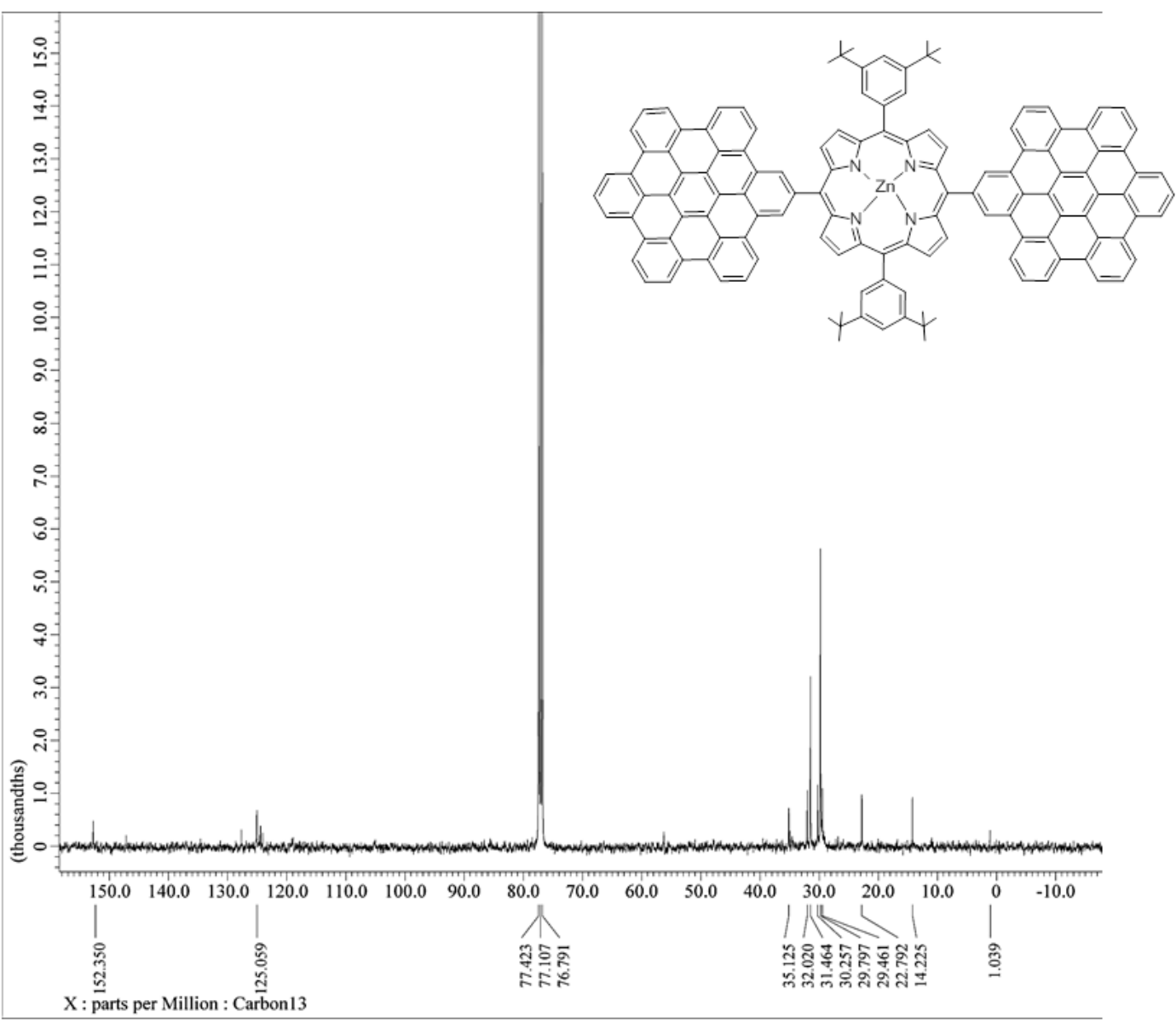

Figure S10. ${ }^{13} \mathrm{C}-\mathrm{NMR}$ spectra of $\mathbf{1 d}$ porphyrin in $\mathrm{CDCl}_{3}$. 


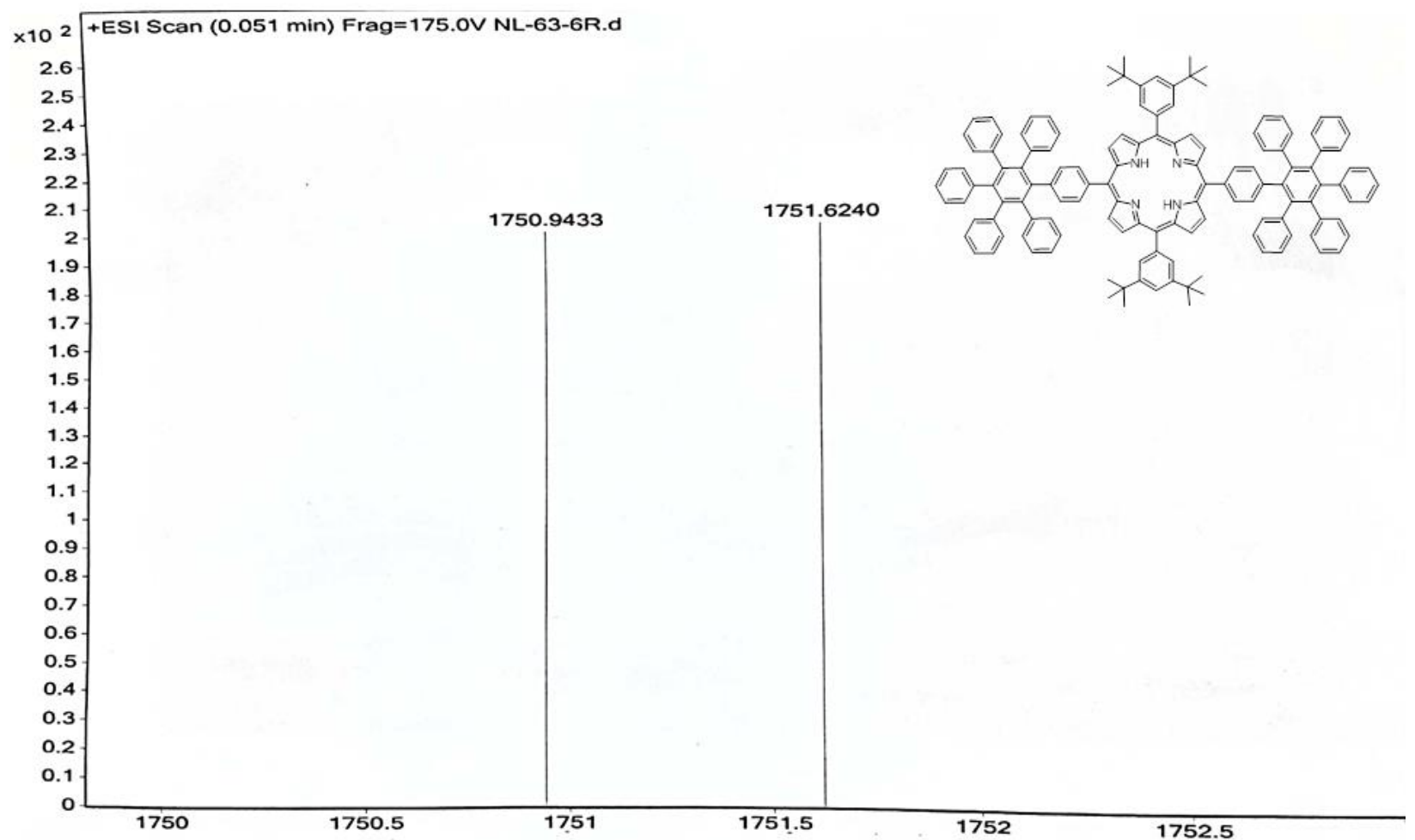

Figure S11. Mass Spectra of 1a porphyrin. 


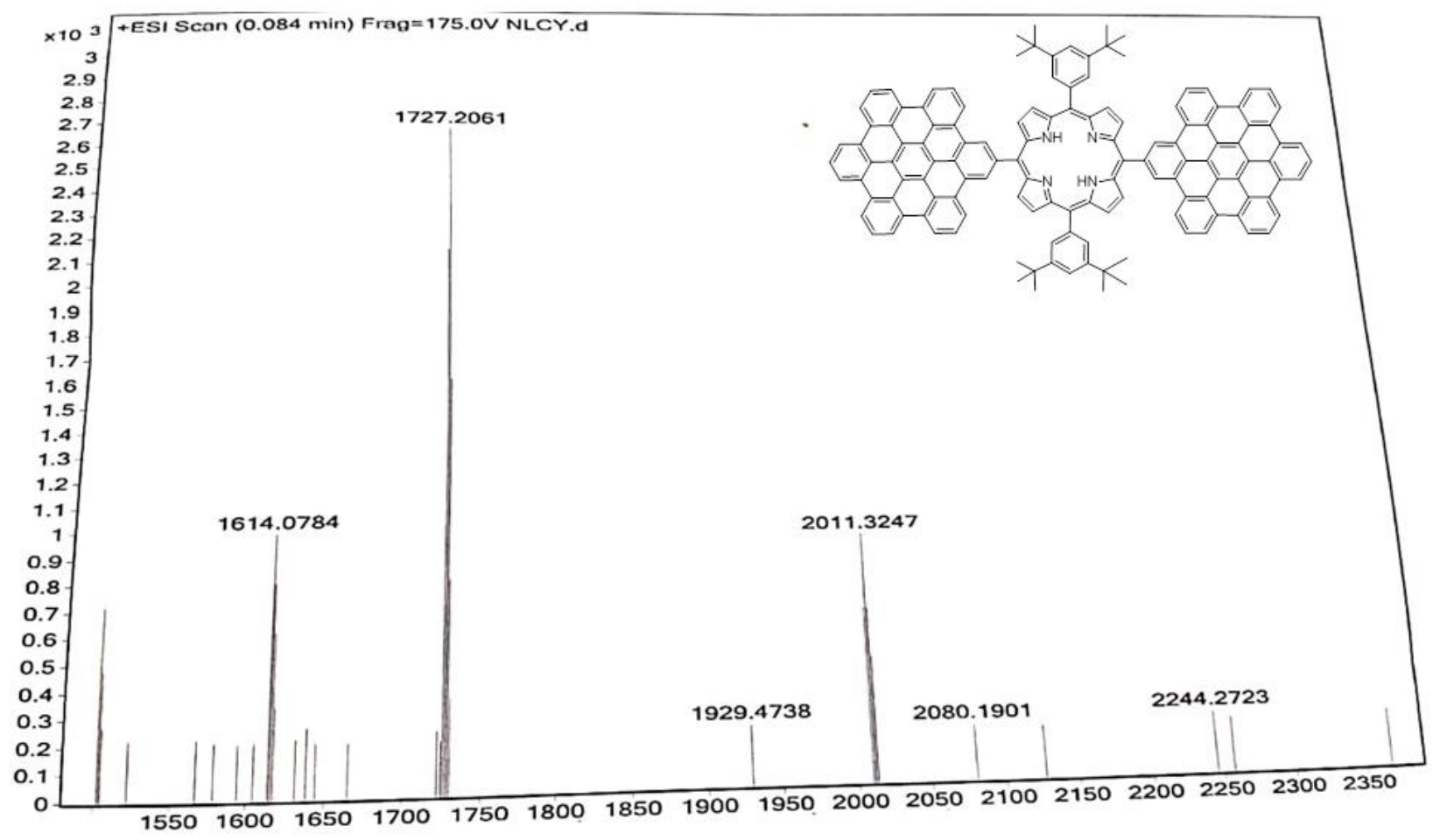

Figure S12. Mass Spectra of $\mathbf{1 b}$ porphyrin. 


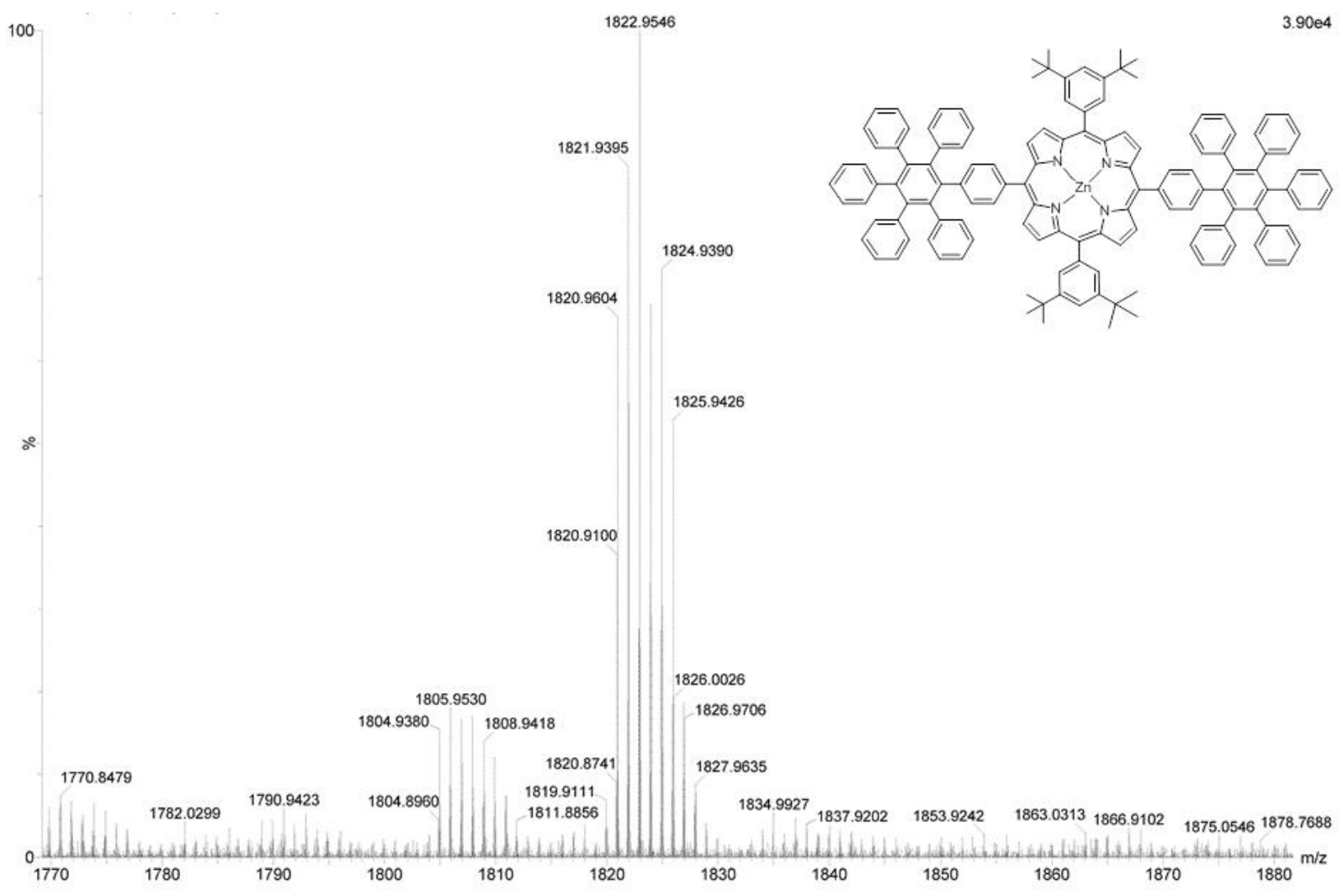

Figure S13. Mass Spectra of 1c porphyrin. 


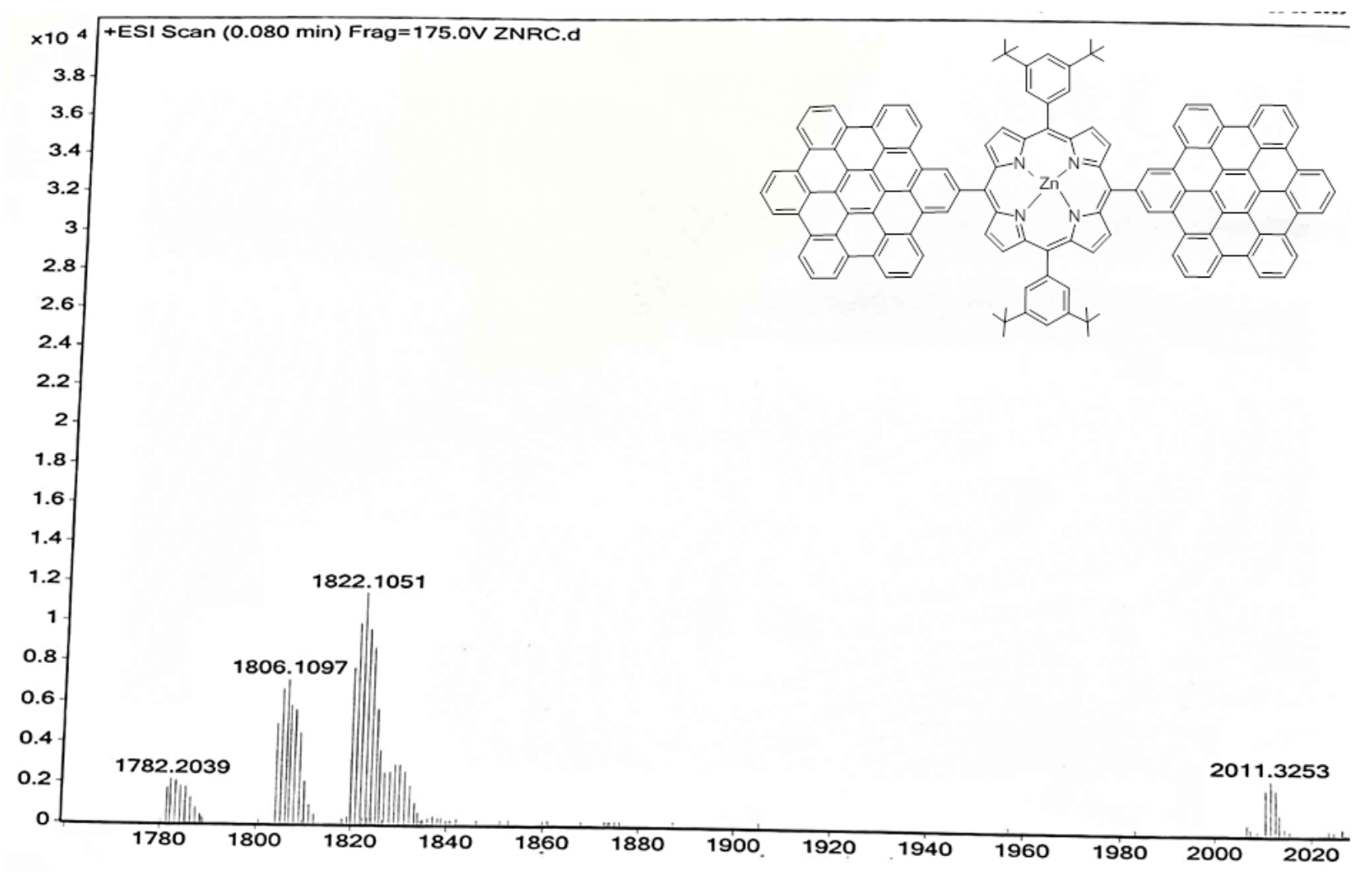

Figure S14. Mass Spectra of 1d porphyrin in $\mathrm{CDCl}_{3}$.
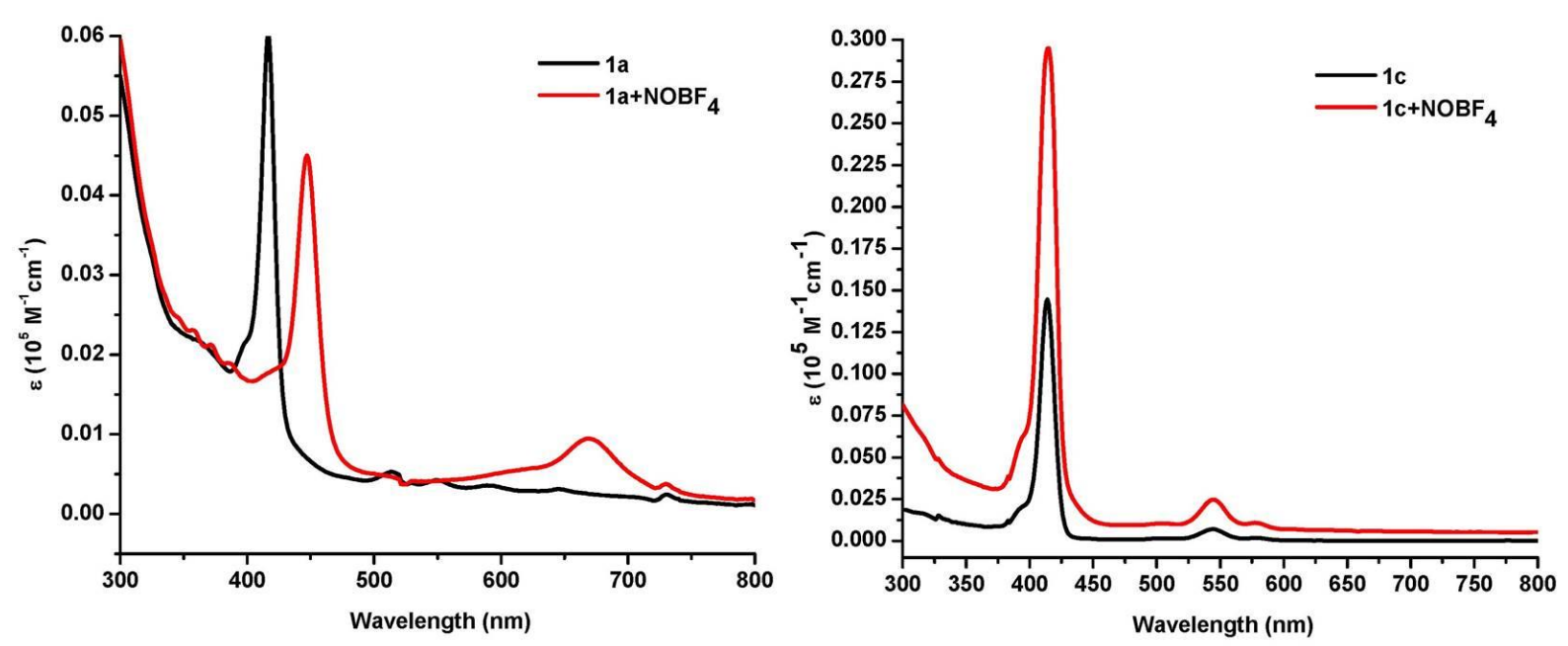

Figure S15. UV-Vis spectra of 1a (left) and 1c (right) in DCM before and after addition of $\mathrm{NOBF}_{4}\left(5 \times 10^{-5} \mathrm{M}\right)$. 

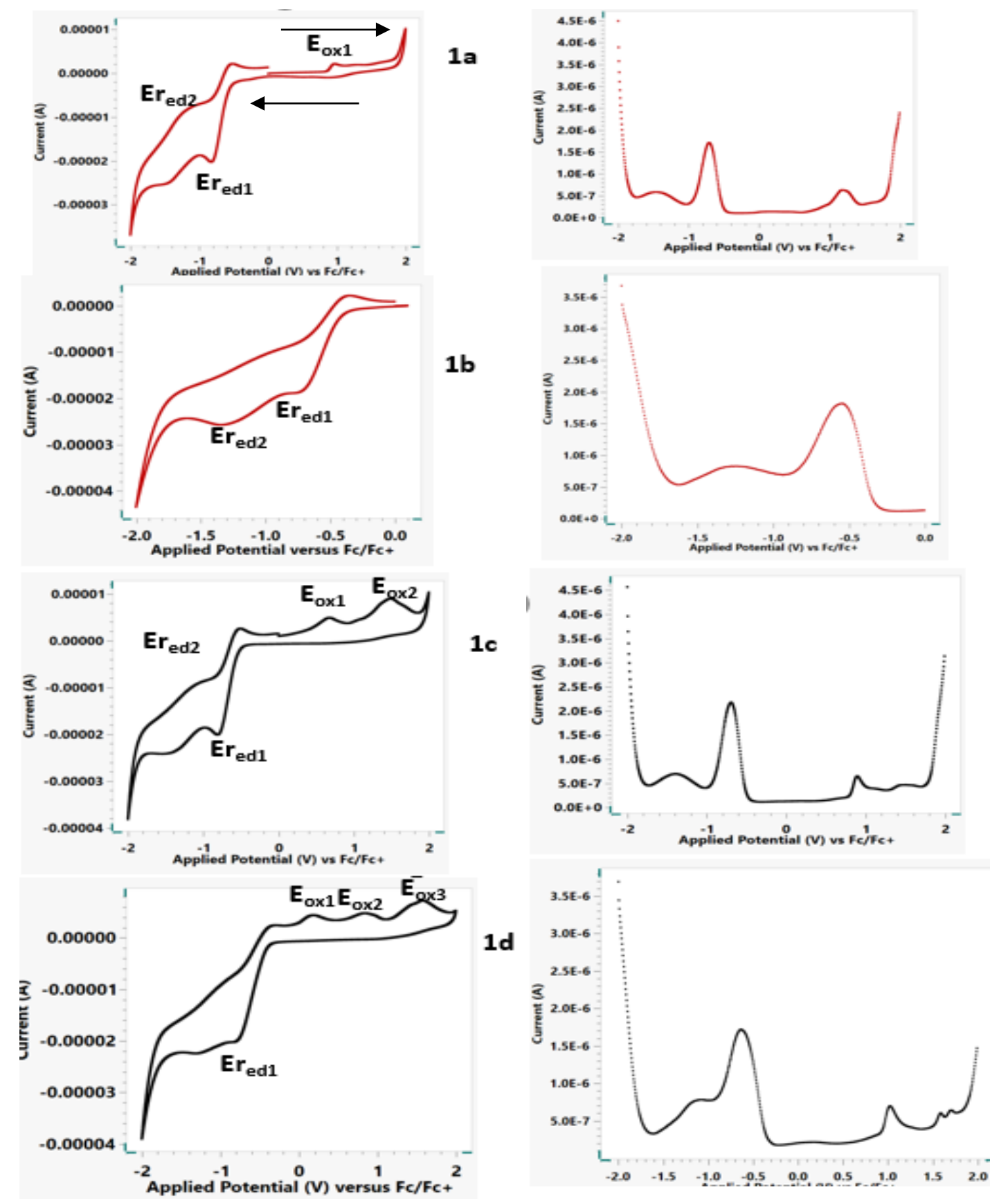

Figure S16. Cyclic Voltammogram and differential pulse voltammogram (DPV) plots of 1a, 1b, 1c and 1d, in $\left(5 \times 10^{-5} \mathrm{M}\right) \mathrm{CH}_{2} \mathrm{Cl}_{2}$ containing $0.1 \mathrm{M} \mathrm{nBu}_{4} \mathrm{NPF}_{6}$ as a supporting electrolyte versus the ferrocene/ferrocenium cation. Scan rate=0.1v/s; working electrode $\mathrm{Pt}$; counter electrode $=$ glassy carbon; reference electrode $=\mathrm{Ag} / \mathrm{AgCl}$ in $\mathrm{DCM}$. The direction of current is from left to right for all the CV graphs. 

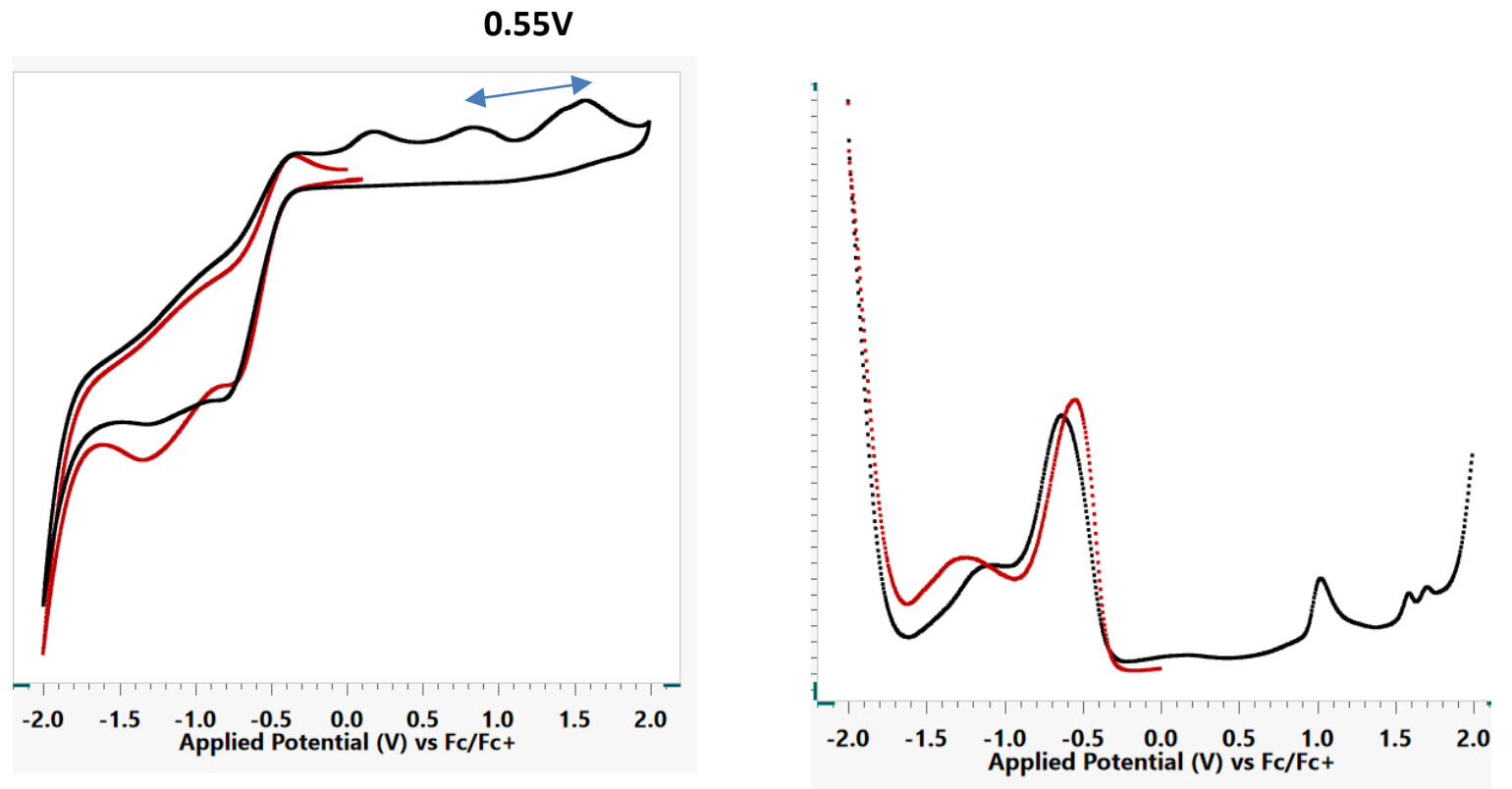

Figure S17. Overlayed graphs depicting changes in oxi/red peaks in $\mathbf{1 b}$ and $\mathbf{1 d .}$

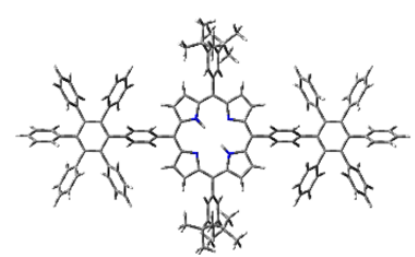

(a)

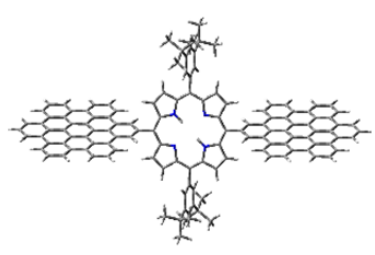

(b)

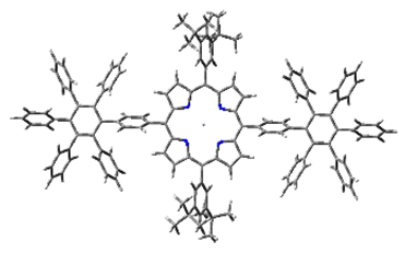

(c)

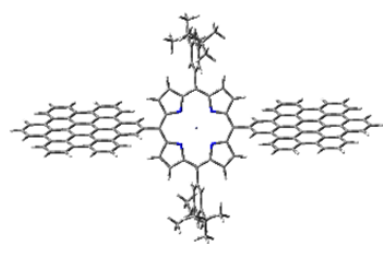

(d)

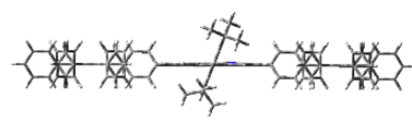

(e)

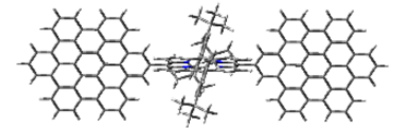

(f)

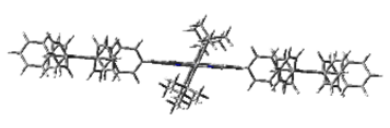

(g)

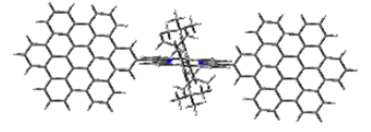

(h)

Figure S18. Ground state optimized structure of 1a ((a) top view and (e) side view); 1b ((b) top view and (f) side view); 1c ((c) top view and (g) side view); 1d ((d) top view and (h) side view). 

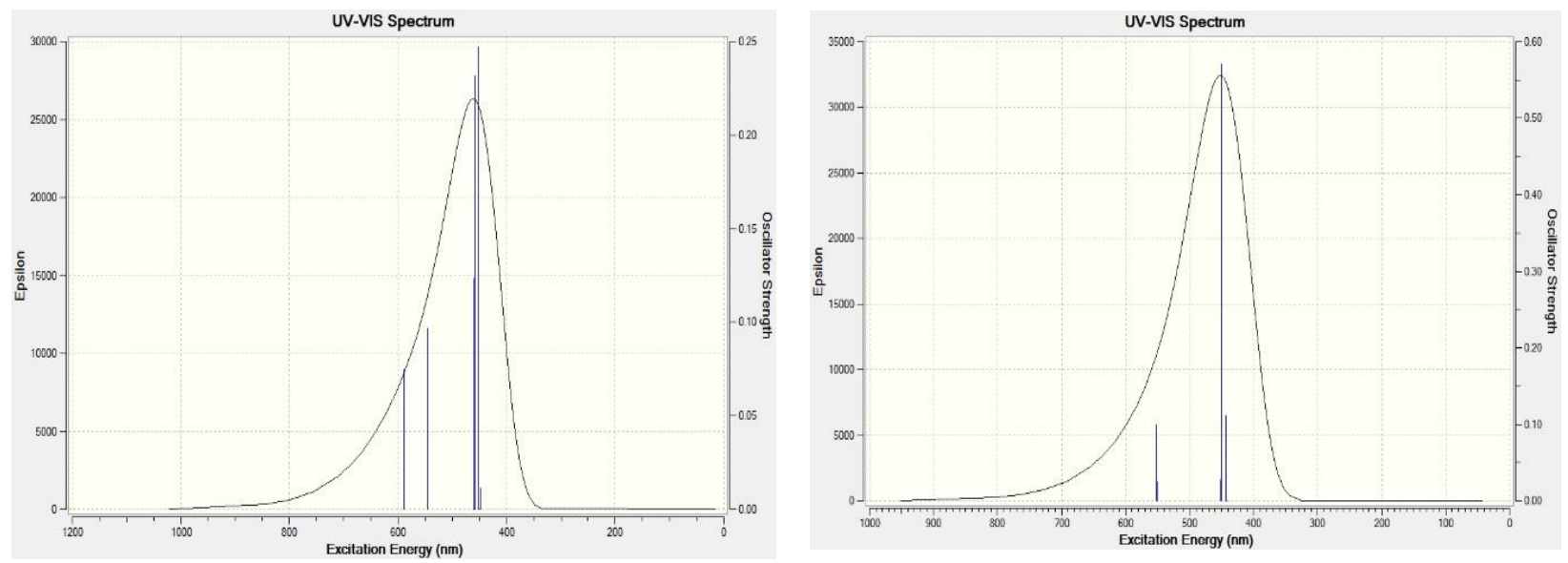

Figure S19. Time dependent DFT based absorption spectra of $\mathbf{1 b}$ (left) and $\mathbf{1 d}$ (right).

\section{FMO analysis}

Table S1: Calculated HOMO, LUMO, band gap $(\Delta \mathrm{E})$ energies, chemical hardness $(\eta)$ and chemical potential $(\mu)$ of $\mathbf{1 a}, \mathbf{1 a}^{+\cdot}, \mathbf{1 b}, \mathbf{1 c}, \mathbf{1} \mathbf{c}^{+\cdot}$ and $\mathbf{1 d}$.

\begin{tabular}{|c|c|c|c|c|c|}
\hline & $\begin{array}{c}\text { HOMO } \\
(\mathrm{eV})\end{array}$ & $\begin{array}{c}\text { LUMO } \\
(\mathbf{e V})\end{array}$ & $\begin{array}{c}\text { Band gap } \\
(\Delta E)\end{array}$ & $\begin{array}{c}\eta \\
(\mathrm{eV})\end{array}$ & $\begin{array}{c}\boldsymbol{\mu} \\
(\mathrm{eV})\end{array}$ \\
\hline $1 \mathrm{a}$ & -4.88 & -2.27 & 2.61 & 1.30 & 3.58 \\
\hline $\mathbf{1 a}^{+\cdot}$ & -7.31 & -5.16 & 2.15 & 1.07 & 6.24 \\
\hline $1 b$ & -5.07 & -2.46 & 2.61 & 1.30 & 3.76 \\
\hline 1c & -4.91 & -2.20 & 2.71 & 1.35 & 3.56 \\
\hline $1 c^{+\cdot}$ & -7.31 & -5.00 & 2.31 & 1.15 & 6.16 \\
\hline 1d & -5.10 & -2.40 & 2.70 & 1.35 & 3.75 \\
\hline
\end{tabular}

Note: The values of the chemical potential $(\mu)$ and chemical hardness $(\eta)$ of synthesized porphyrins and their radical cations were calculated using the equation, $\mu=\left(E_{\text {номо }}+\right.$ $\left.E_{L U M O}\right) / 2$, and $\eta=\left(E_{L U M O}-E_{\text {HOMO }}\right) / 2$, respectively.

From the frontier molecular orbital analysis, we can deduce the chemical hardness $(\eta)$. Greater the value of chemical hardness, greater will be the stability of the molecule. From Table S1, it 
can be inferred that the chemical hardness decreases as electrons are removed from the porphyrin to form radical cation.

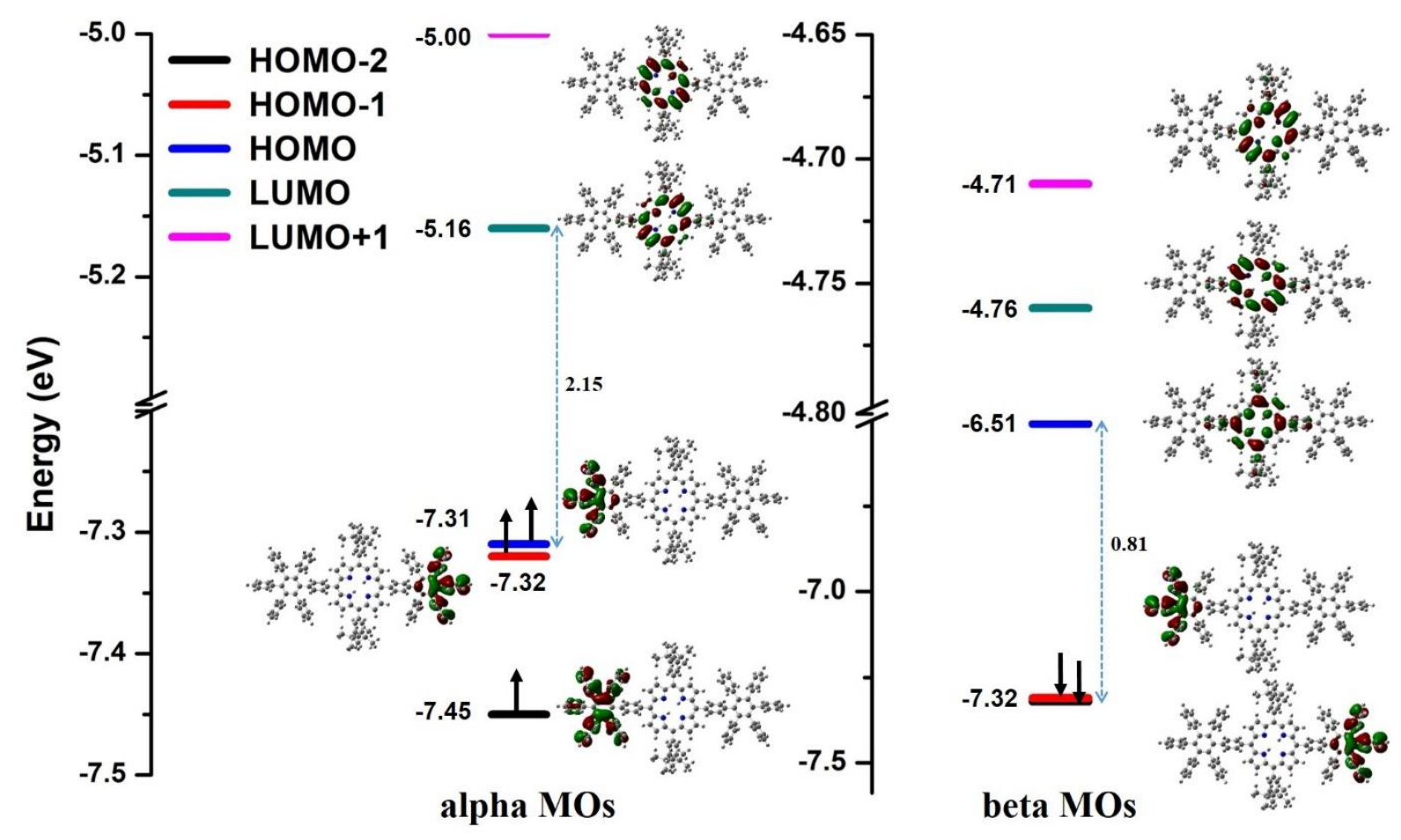

Figure S20. Selected molecular orbitals of radical cation of 1 a porphyrin calculated at B3LYP/LANL2DZ (C, H, N,) level. 


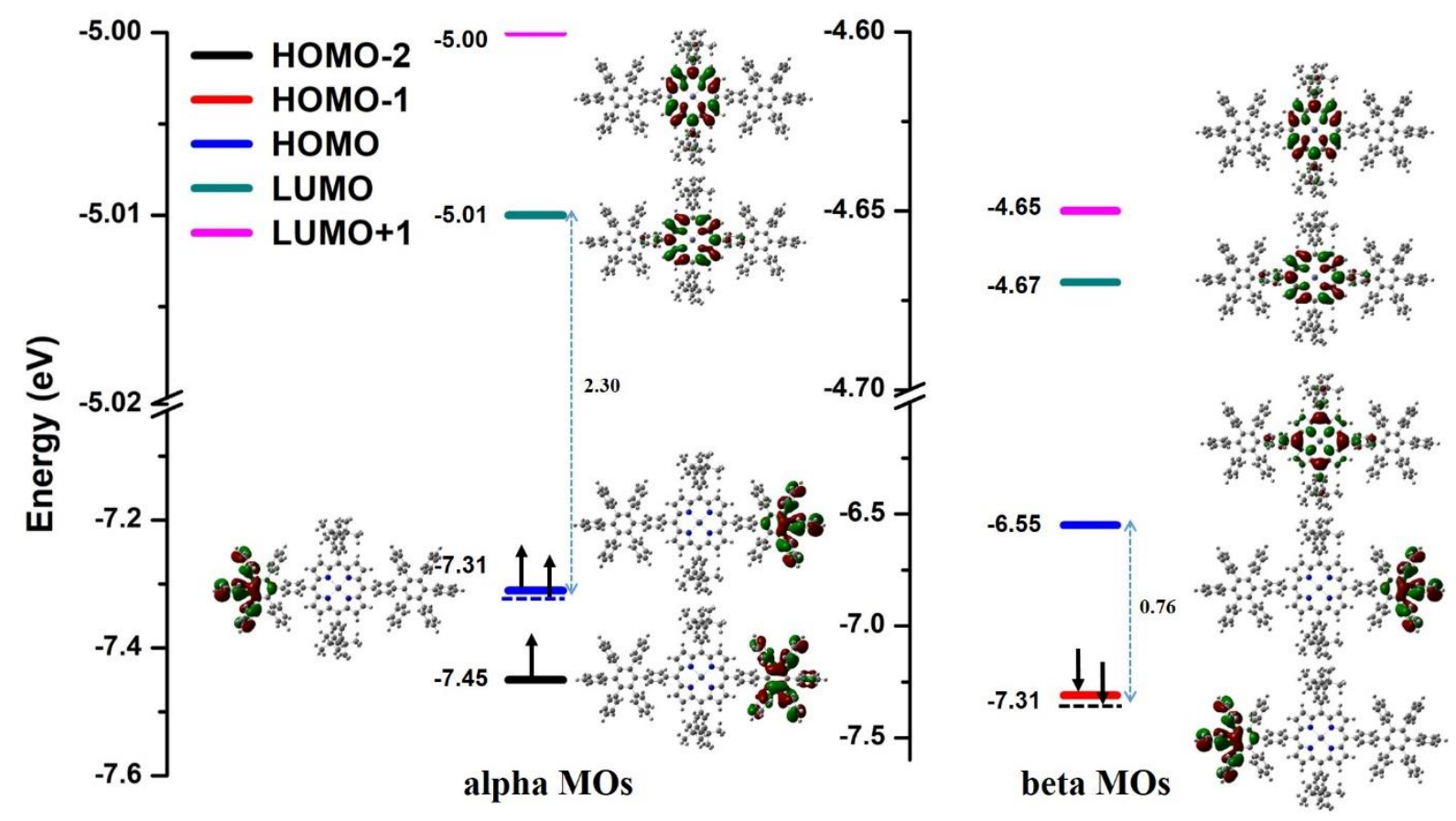

Figure S21. Selected molecular orbitals of radical cation of 1c porphyrin calculated at B3LYP/LANL2DZ (C, H, N, Zn) level. 
Table S2. Calculated Mulliken, Natural and ESP charges and Spin densities of (1a) and (1a ${ }^{+}$) of porphyrin.

\begin{tabular}{|c|c|c|c|c|c|c|c|}
\hline & \multicolumn{3}{|c|}{$1 \mathbf{a}$} & \multicolumn{4}{|c|}{$1 a^{+\cdot}$} \\
\hline Atom & ESP & Natural & Mulliken & ESP & Natural & Mulliken & Spin density \\
\hline $\mathrm{N}(5,18)$ & -0.34 & -0.57 & -0.43 & -0.32 & -0.54 & -0.39 & 0.07 \\
\hline $\mathrm{N}$ (amine)(12,24) & -0.58 & -0.55 & -0.20 & -0.54 & -0.58 & -0.20 & 0.21 \\
\hline $\mathrm{H}(123,128)$ & 0.23 & 0.48 & 0.43 & 0.22 & 0.48 & 0.43 & -0.01 \\
\hline $\mathrm{C}(6,7,13,19)$ & 0.54 & 0.05 & 0.15 & 0.30 & 0.03 & 0.16 & 0.23 \\
\hline $\mathrm{C}(1,4,14,17)$ & 0.46 & 0.22 & 0.13 & 0.36 & 0.20 & 0.14 & -0.03 \\
\hline $\mathrm{C}(8,11,20,23)$ & 0.61 & 0.18 & -0.01 & 0.48 & 0.18 & -0.04 & -0.09 \\
\hline $\mathrm{C}(26,27)$-ligand & 0.32 & -0.09 & 0.25 & 0.27 & -0.07 & 0.25 & -0.02 \\
\hline $\begin{array}{l}\mathrm{H} \text { (beta pyrollic } \\
\text { protons) }\end{array}$ & 0.23 & 0.24 & 0.39 & 0.21 & 0.25 & 0.29 & 0.00 \\
\hline
\end{tabular}




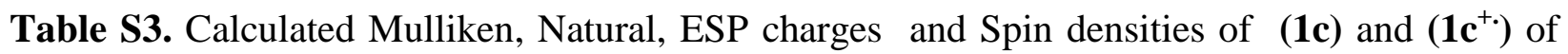
porphyrin.

\begin{tabular}{|c|c|c|c|c|c|c|c|}
\hline & \multicolumn{3}{|c|}{ 1c } & \multicolumn{3}{c|}{ 1c $^{+.}$} \\
\hline Atom & ESP & Natural & Mulliken & ESP & Natural & Mulliken & Spin density \\
\hline $\mathrm{N}(5,18)$ & -0.98 & -0.72 & -0.45 & -0.92 & -0.69 & -0.44 & 0.10 \\
\hline $\mathrm{N}(12,24)$ & -0.97 & -0.72 & -0.45 & -0.90 & -0.69 & -0.44 & 0.10 \\
\hline $\mathrm{Zn}$ & 1.36 & 1.38 & 1.08 & 1.36 & 1.42 & 1.12 & 0.01 \\
\hline $\mathrm{C}(6,7,13,19)$ & -0.61 & -0.052 & 0.12 & -0.42 & 0.04 & 0.15 & 0.07 \\
\hline $\begin{array}{c}\mathrm{C}(1,4,14,17) \\
\mathrm{C}(8,11,20,23)\end{array}$ & 0.72 & 0.20 & 0.12 & 0.65 & 0.19 & 0.11 & 0.24 \\
\hline $\begin{array}{c}\mathrm{C}(26,27)- \\
\text { ligand }\end{array}$ & 0.34 & -0.04 & 0.26 & 0.16 & -0.07 & 0.28 & -0.02 \\
\hline $\begin{array}{c}\mathrm{H}(\text { betapyrollic } \\
\text { protons })\end{array}$ & 0.23 & 0.23 & 0.27 & 0.22 & 0.24 & 0.28 & 0.00 \\
\hline
\end{tabular}

Table S4. Calculated electronic excitation energies and corresponding oscillator strengths of the low-lying singlet excited states of $\mathbf{1 b}$ and $\mathbf{1 d}$.

\begin{tabular}{|c|l|c|c|c|c|}
\hline Porphyrin & $\begin{array}{c}\text { Electronic } \\
\text { transition }\end{array}$ & $\begin{array}{c}\text { Energy } \\
(\mathbf{n m})\end{array}$ & $\begin{array}{c}\text { Oscillatory } \\
\text { Strength (f) }\end{array}$ & Contribution & CI \\
\hline $\mathbf{1 b}$ & $\mathrm{S}_{0} \rightarrow \mathrm{S}_{1}$ & 588.70 & 0.0748 & $\mathrm{HOMO} \rightarrow$ LUMO & 0.575 \\
& & & & & \\
\hline & $\mathrm{S}_{0} \rightarrow \mathrm{S}_{2}$ & 545.75 & 0.0967 & $\mathrm{HOMO} \rightarrow \mathrm{LUMO}+1$ & 0.557 \\
\hline
\end{tabular}




\begin{tabular}{|c|c|c|c|c|c|}
\hline & $\mathrm{S}_{0} \rightarrow \mathrm{S}_{3}$ & 461.10 & 0.1235 & HOMO- $1 \rightarrow$ LUMO & 0.459 \\
\hline & $\mathrm{S}_{0} \rightarrow \mathrm{S}_{4}$ & 460.52 & 0.0000 & $\mathrm{HOMO}-3 \rightarrow \mathrm{LUMO}+1$ & 0.635 \\
\hline & $\mathrm{S}_{0} \rightarrow \mathrm{S}_{5}$ & 458.78 & 0.0000 & HOMO- $2 \rightarrow$ LUMO & 0.673 \\
\hline & $\mathrm{S}_{0} \rightarrow \mathrm{S}_{6}$ & 458.32 & 0.2320 & HOMO-4 $\rightarrow$ LUMO & 0.565 \\
\hline & $\mathrm{S}_{0} \rightarrow \mathrm{S}_{7}$ & 451.96 & 0.2473 & $\mathrm{HOMO}-1 \rightarrow \mathrm{LUMO}+1$ & 0.448 \\
\hline & $\mathrm{S}_{0} \rightarrow \mathrm{S}_{8}$ & 450.18 & 0.0000 & $\mathrm{HOMO}-2 \rightarrow \mathrm{LUMO}+1$ & 0.668 \\
\hline & $\mathrm{S}_{0} \rightarrow \mathrm{S}_{9}$ & 449.39 & 0.0000 & $\mathrm{HOMO}-3 \rightarrow \mathrm{LUMO}+1$ & 0.653 \\
\hline & $\mathrm{S}_{0} \rightarrow \mathrm{S}_{10}$ & 448.94 & 0.0115 & $\mathrm{HOMO}-4 \rightarrow \mathrm{LUMO}+1$ & 0.638 \\
\hline 1d & $\mathrm{S}_{0} \rightarrow \mathrm{S}_{1}$ & 552.68 & 0.0996 & $\mathrm{HOMO} \rightarrow \mathrm{LUMO}$ & 0.569 \\
\hline & $\mathrm{S}_{0} \rightarrow \mathrm{S}_{2}$ & 549.77 & 0.0247 & $\mathrm{HOMO} \rightarrow \mathrm{LUMO}+1$ & 0.554 \\
\hline & $\mathrm{S}_{0} \rightarrow \mathrm{S}_{3}$ & 452.28 & 0.0000 & HOMO-3 $\rightarrow$ LUMO & 0.631 \\
\hline & $\mathrm{S}_{0} \rightarrow \mathrm{S}_{4}$ & 452.06 & 0.0273 & HOMO-5 $\rightarrow$ LUMO & 0.541 \\
\hline
\end{tabular}




\begin{tabular}{|c|c|c|c|c|}
\hline $\mathrm{S}_{0} \rightarrow \mathrm{S}_{5}$ & 450.63 & 0.5701 & HOMO-4 $\rightarrow$ LUMO & 0.592 \\
\hline $\mathrm{S}_{0} \rightarrow \mathrm{S}_{6}$ & 449.54 & 0.0000 & HOMO-3 $\rightarrow$ LUMO & 0.688 \\
\hline $\mathrm{S}_{0} \rightarrow \mathrm{S}_{7}$ & 443.46 & 0.1115 & HOMO-5 $\rightarrow$ LUMO +1 & 0.424 \\
\hline $\mathrm{S}_{0} \rightarrow \mathrm{S}_{8}$ & 443.19 & 0.0692 & $\mathrm{HOMO}-4 \rightarrow \mathrm{LUMO}+1$ & 0.497 \\
\hline $\mathrm{S}_{0} \rightarrow \mathrm{S}_{9}$ & 443.10 & 0.0000 & $\mathrm{HOMO}-2 \rightarrow \mathrm{LUMO}+1$ & 0.644 \\
\hline $\mathrm{S}_{0} \rightarrow \mathrm{S}_{10}$ & 441.75 & 0.0000 & $\mathrm{HOMO}-3 \rightarrow \mathrm{LUMO}+1$ & 0.681 \\
\hline
\end{tabular}




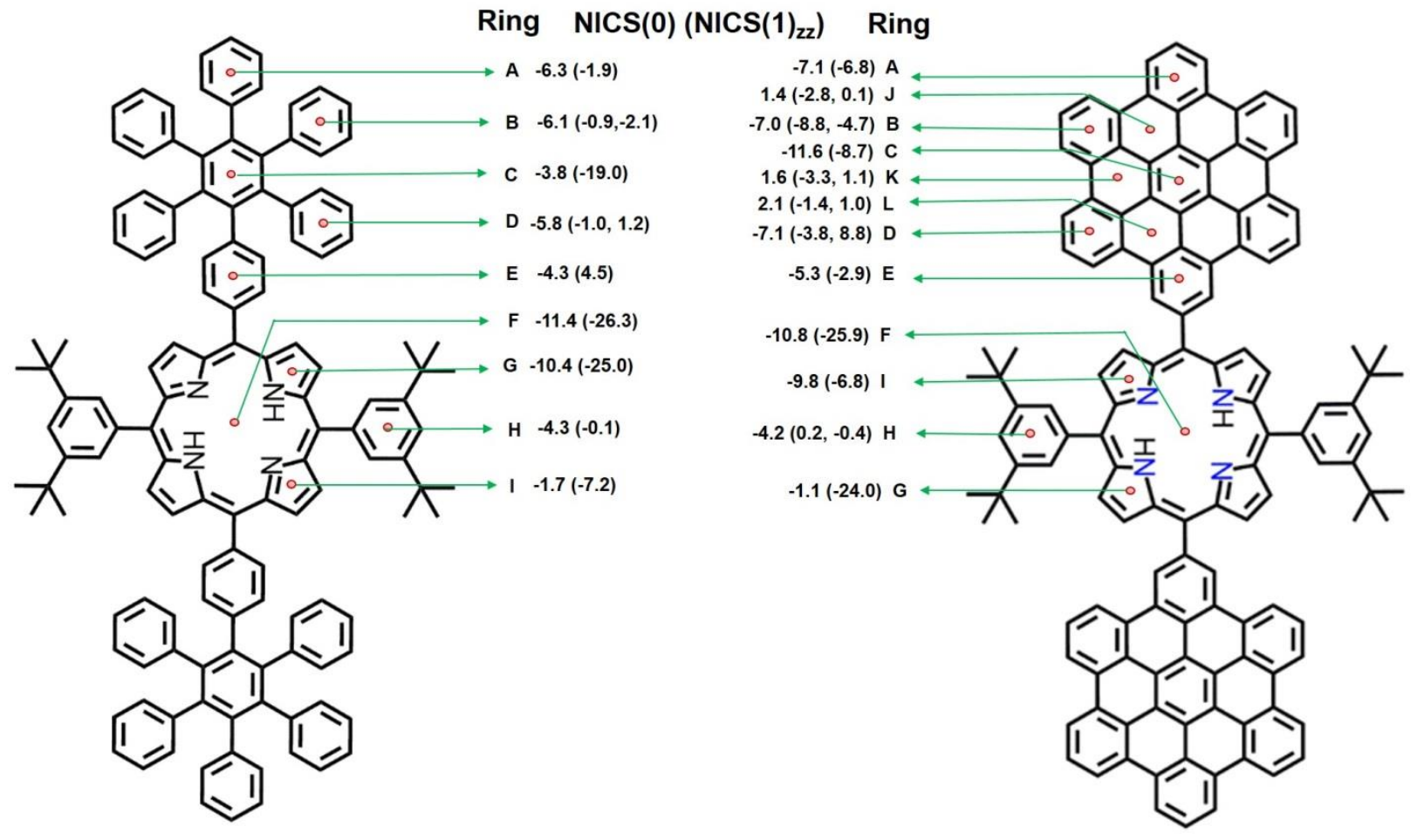

Figure S22. $\mathrm{NICS}(0)$ and $\mathrm{NICS}(1)_{\mathrm{zz}}$ values of $\mathbf{1 a}$ (left) and $\mathbf{1 b}$ (right) calculated by GIAO/DFT/LANL2DZ level of theory

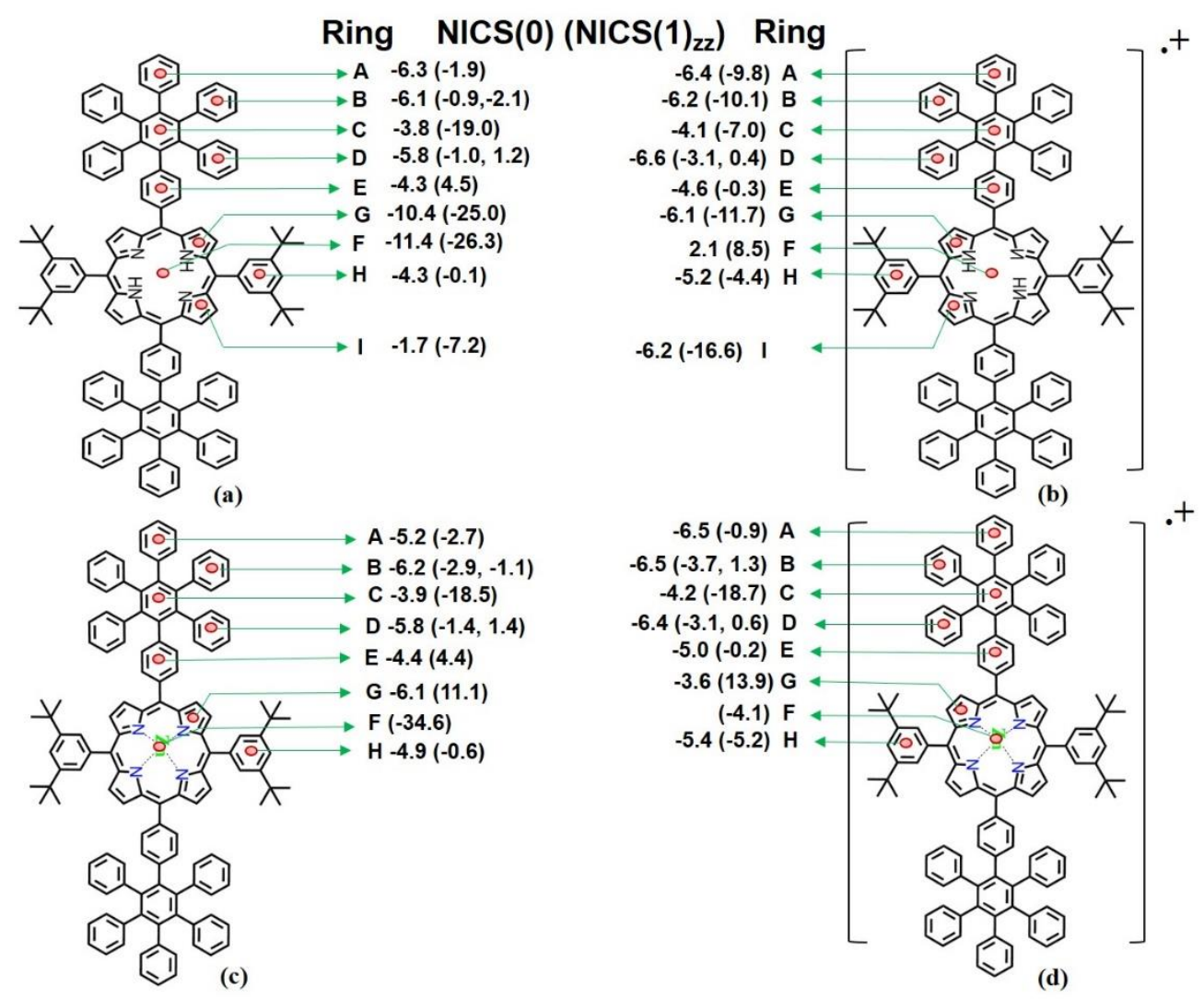

Figure S23. NICS Values of (a) 1a, (b) $1 \mathbf{a}^{+\cdot}$, and (c) $1 \mathbf{c}$ and (d) $1 \mathbf{c}^{+\cdot}$ 